\title{
Piperlongumine induces autophagy by targeting p38 signaling
}

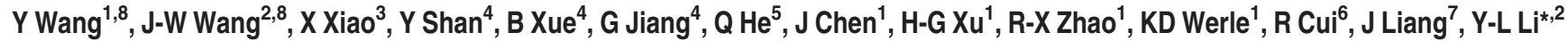 \\ and $\mathrm{Z}-\mathrm{X} X \mathrm{U}^{*, 1}$
}

Piperlongumine (PL), a natural product isolated from the plant species Piper longum L., can selectively induce apoptotic cell death in cancer cells by targeting the stress response to reactive oxygen species (ROS). Here we show that PL induces cell death in the presence of benzyloxycarbonylvalyl-alanyl-aspartic acid (0-methyl)-fluoro-methylketone (zVAD-fmk), a pan-apoptotic inhibitor, and in the presence of necrostatin-1, a necrotic inhibitor. Instead PL-induced cell death can be suppressed by 3-methyladenine, an autophagy inhibitor, and substantially attenuated in cells lacking the autophagy-related 5 (Atg5) gene. We further show that PL enhances autophagy activity without blocking autophagy flux. Application of $\mathrm{N}$-acetyl-cysteine, an antioxidant, markedly reduces PL-induced autophagy and cell death, suggesting an essential role for intracellular ROS in PL-induced autophagy. Furthermore, PL stimulates the activation of $\mathrm{p} 38$ protein kinase through ROS-induced stress response and p38 signaling is necessary for the action of PL as SB203580, a p38 inhibitor, or dominant-negative p38 can effectively reduce PL-mediated autophagy. Thus, we have characterized a new mechanism for PL-induced cell death through the ROS-p38 pathway. Our findings support the therapeutic potential of PL by triggering autophagic cell death.

Cell Death and Disease (2013) 4, e824; doi:10.1038/cddis.2013.358; published online 3 October 2013

Subject Category: Cancer

Autophagy is a regulated lysosomal pathway involved in the degradation and recycling of long-lived proteins and organelles within cells. During autophagy, cytoplasmic constituents are sequestered into double-membraned autophagosomes, which then fuse with lysosomes to form autolysosomes, in which degradation occurs. Through the catabolic metabolism, autophagy digests cellular contents to provide cellular energy and building blocks for biosynthesis. ${ }^{1-6}$ In addition to its basic role in the turnover of proteins and organelles, autophagy possesses multiple physiological and pathophysiological functions in the maintenance of cellular homeostasis and in the response to stresses. $^{7-15}$

Consistent with the notion that autophagy has an adaptive role under cellular stress conditions, some researchers showed that autophagy may act in a positive way for malignant progression and in subsequent tumor maintenance. Although the phenomena are frequently observed in established neoplastic lesions and much needs to be learned about the regulation and context-dependent biological functions of autophagy, there are many reports that show autophagy may act as a tumor suppressor, in particular in the initiation of tumor. ${ }^{16-24}$ Keeping up with this concept, studies have shown that induction of autophagy becomes an alternative strategy for cancer treatment. ${ }^{25}$ Many therapeutic drugs or natural compounds can trigger caspase-independent autophagic cell death by activating autophagy signaling pathways. ${ }^{25,26}$

Piperlongumine (PL) is an alkaloid isolated from the long pepper (Piper longum L.) possessing anti-platelet aggregation, anti-inflammatory and anticancer properties. Raj et al. ${ }^{27}$ recently found that $\mathrm{PL}$ is the strongest activator of p53-mediated transcription among several thousand biologically active compounds. In a melanoma allografted mouse model, the antitumor effect of PL was greater than that of the cytotoxic chemotherapeutic drug, cisplatin. Similarly, PL outperformed paclitaxel in controlling a polyoma middle $\mathrm{T}$ antigen-driven mouse model of spontaneous mammary tumorigenesis. ${ }^{28}$

\footnotetext{
${ }^{1}$ Division of Hematology and Oncology, Comprehensive Cancer Center, Department of Medicine, University of Alabama at Birmingham, Birmingham, AL, USA; ${ }^{2}$ Key Laboratory of Pathobiology, Ministry of Education, Norman Bethune College of Medicine, Jilin University, Changchun, China; ${ }^{3}$ Department of Chemistry, Central South University, Hunan, China; ${ }^{4}$ Department of Surgery, Second Affiliated Hospital, Soochow University, Suzhou, Jiangsu, China; ${ }^{5}$ Department of Pathology, Xiangya Hospital, Central South University, Hunan, China; ${ }^{6}$ Department of Dermatology, Boston University, School of Medicine, Boston, MA, USA and ${ }^{7}$ Department of Systems Biology, UT MD Anderson Cancer Center, Houston, TX, USA

*Corresponding authors: Y-L Li, Key Laboratory of Pathobiology, Ministry of Education, Norman Bethune College of Medicine, Jilin University, No. 126, Xin-Min Avenue, Changchun, Jilin, China. Tel: +86 431 85619481; Fax: +86 431 85619470; E-mail: ylli@jlu.edu.cn

or Z-X Xu, Division of Hematology and Oncology, Comprehensive Cancer Center, Department of Medicine, University of Alabama at Birmingham, 1824 6th Avenue South, Wallace Tumor Institute Building, Room 520D, Birmingham, AL 35205, USA. Tel: +1 205934 1868; Fax: +1 205 934 1870; E-mail: zhixiangxu@uabmc.edu ${ }^{8}$ These authors contributed equally to this work.

Keywords: piperlongumine; autophagy; p38; reactive oxygen species

Abbreviations: AMPK, AMP-activated protein kinase; ATF-2, activating transcription factor 2; Atg5, autophagy-related gene-5; Baf-A1, bafilomycin-A1; CQ, chloroquine; GPX1, glutathione peroxidase 1; LC3, light-chain 3; 3-MA, 3-methyladenine; MAPK, mitogen-activated protein kinase; MAPKAPK-2, MAP kinaseactivated protein kinase 2; MKKs, MAP kinase kinases; MP, methyl pyruvate; MSK1, mitogen- and stress-activated protein kinase 1; NAC, N-acetyl-cysteine; Nec-1, necrostatin-1; PGAM5, phosphoglycerate mutase family member 5; PL, piperlongumine; RIP, receptor-interacting protein; ROS, reactive oxygen species; TTFA, thenoyltrifluoroacetone; zVAD-fmk, benzyloxycarbonylvalyl-alanyl-aspartic acid (0-methyl)-fluoro-methylketone

Received 29.5.13; revised 07.7.13; accepted 11.7.13; Edited by GM Fimia
} 
Raj et $a .^{27}$ found that proteins, such as glutathione-Stransferase-P1 and carbonyl reductase 1 that are involved in the cellular response to oxidative stress were the most enriched group after PL treatment, suggesting a mechanism based on reactive oxygen species (ROS). Indeed, ROSsensitive fluorescent probes revealed that $\mathrm{PL}$ treatment increased ROS levels in cancer cell lines but not in the untransformed cells. Increased ROS levels during cellular transformation imply a cellular adaptation to greater oxidative stress accompanying tumorigenesis. Therefore, cancer cells may have an enhanced reliance on this stress response and might be more susceptible than normal cells to further elevation of intracellular ROS levels by pharmacological intervention, such as PL treatment. ${ }^{27-29}$ In this study, we demonstrated that PL induces active autophagy and non-apoptotic cell death in a manner that is dependent on intracellular ROS and the activation of the p38 pathway.

\section{Results}

PL prompts non-apoptotic and non-necrotic cell death. $\mathrm{PL}$ has been reported to induce apoptotic programmed cell death in vitro and in vivo. To determine whether $\mathrm{PL}$ is able to trigger non-apoptotic cell death, human osteosarcoma cells U2OS were treated with benzyloxycarbonylvalyl-alanyl-aspartic acid (O-methyl)-fluoro-methylketone (zVAD-fmk), a panapoptotic inhibitor, before incubation with PL. As expected, $\mathrm{PL}$ induced the activation of caspase-7, which was markedly decreased by pre-treatment with zVAD-fmk (Figure 1a). However, the cells treated with $\mathrm{PL}$ in the presence of zVAD-fmk for up to $72 \mathrm{~h}$ still underwent cell death although the extent of cell death in zVAD-fmk-treated cells was less than that observed in the cells treated with PL alone (Figures $1 \mathrm{~b}$ and c). To determine whether the PL-induced non-apoptotic cell death is prompted by necrosis, we detected receptor-interacting protein (RIP) kinases RIP1, RIP3 and phosphoglycerate mutase family member 5 (PGAM5) in PL-treated cells, which have been reported to be critical constituents triggering programmed necrosis (necroptosis). ${ }^{30,31} \mathrm{PL}$ could not influence the expression of RIP1, RIP3 and PGAM5 (Figure 1d). Further, PL-associated cell death or cell viability was not affected by the pre-treatment with necrostatin-1 (Nec-1), a selective RIP1 inhibitor ${ }^{32}$ (Figures 1e and f), suggesting that PL does not cause necroptosis. Taken together, we conclude that PL can induce non-apoptotic and non-necrotic cell death.

PL induces autophagy. Autophagy was reported to be associated with non-apoptotic programmed cell death. ${ }^{25,26}$ We asked whether PL-induced non-apoptotic cell death could result from autophagy. To visualize autophagy, we applied U2OS/green fluorescent protein (GFP)-light-chain 3 (LC3) cells, a stable cell line expressing GFP-tagged LC3. $^{26,33}$ GFP-LC3 formed cytoplasmic puncta in cells treated with $\mathrm{PL}$ but not in control cells (Figures $2 \mathrm{a}-\mathrm{c}$ and Supplementary Figure S1). zVAD-fmk treatment alone induced very few punctuate GFP-LC3 fluorescent vesicles (Figures 2b and c). Immunoblot showed that LC3 underwent a conversion from the LC3-I isoform to the LC3-II isoform in PL-treated cells, indicating the induction of autophagy
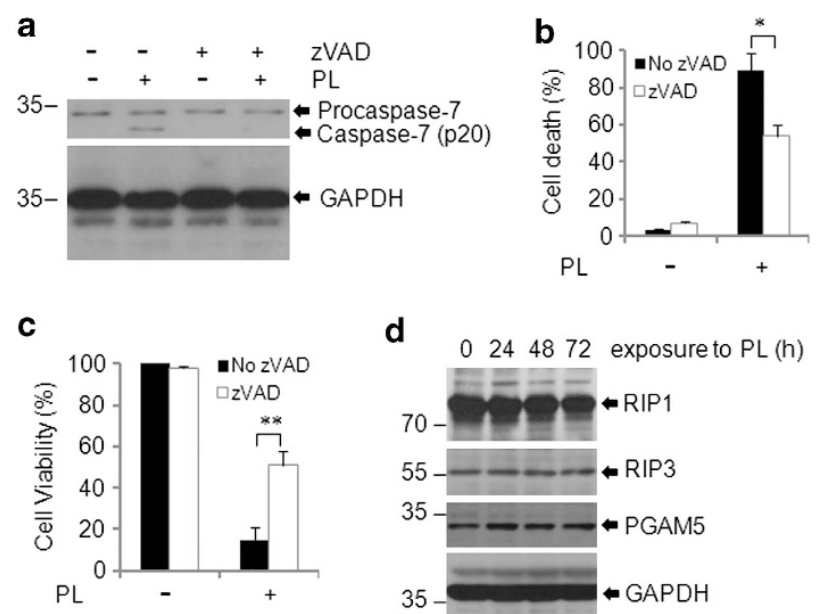

d
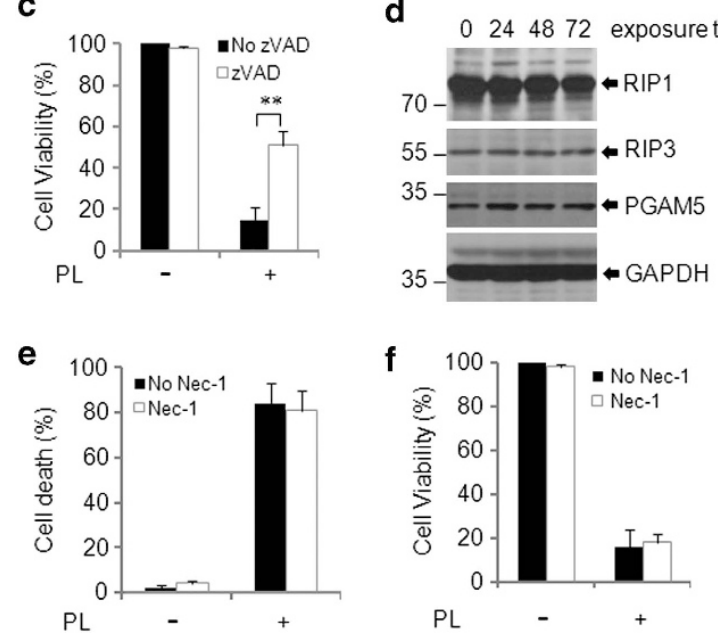

Figure $1 \mathrm{PL}$ induces non-apoptotic and non-necrotic cell death. (a-c) U2OS cells were pre-treated with zVAD $(50 \mu \mathrm{M})$ for $2 \mathrm{~h}$, followed by treatment with PL $(10 \mu \mathrm{M})$ for $72 \mathrm{~h}$. (a) Cleavage of caspase-7 induced by PL and inhibition of the cleavage were analyzed by western blot. (b and $\mathbf{c}$ ) Cell death was determined by Trypan blue exclusion assay and cell viability was measured by the MTT assay. ${ }^{\star} P<0.05$; ${ }^{*} P<0.01$. (d) U2OS cells were treated with $\mathrm{PL}(10 \mu \mathrm{M})$ for the indicated time points. After the treatment, cells were collected and whole-cell extracts (WCE) were isolated for western blot analysis. Thirty micrograms of total proteins were loaded. GAPDH served as a loading control. (e and f) U2OS cells were pre-treated with NEC-1 $(5 \mu \mathrm{M})$ for $2 \mathrm{~h}$, followed by treatment with PL $(10 \mu \mathrm{M})$ for $72 \mathrm{~h}$. Cell death was determined by Trypan blue exclusive assay and cell viability was measured by the MTT assay. Data are shown as mean \pm S.D. $(n=3)$

(Figure 2f). Consistent with the findings in U2OS/GFP-LC3 cells, punctuate mCherry-LC3 signaling was induced in HeLa/mCherry-LC3 cells or wild-type (WT) autophagyrelated gene-5 (ATG5)/mCherry-LC3 mouse embryonic fibroblasts (MEFs) following exposure to $\mathrm{PL}$ in the presence or absence of zVAD-fmk (Figures 2d and e; Supplementary Figure S2).

Electron microscopy revealed the formation of double- or multi-membrane-bound structures containing recognizable cellular organelles and high electron density substance, characteristic of autophagosomes and autolysosomes, in PL-treated U2OS cells (Figure $2 \mathrm{~g}$ ). In contrast, the majority of the nuclei retained normal appearance in PL- and zVAD-fmktreated cells with neither chromatin condensation nor nuclear fragmentation being observed, confirming the absence of apoptotic cell death (Figure $2 \mathrm{~g}$ ). Taken together, our results suggest that PL induces autophagy.

PL does not block autophagy flux. Principally, elevation of autophagy may result from increased autophagy formation or block of autophagy flux. To determine which mechanism is involved in the elevated autophagy in PL-treated cells, we 
a

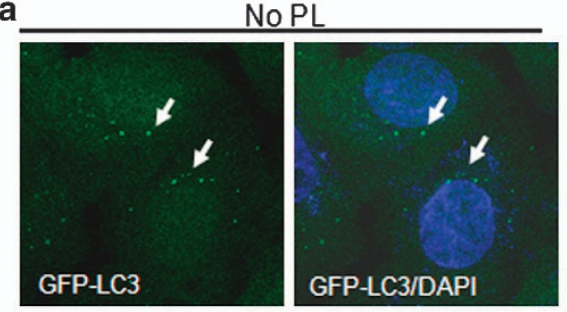

b

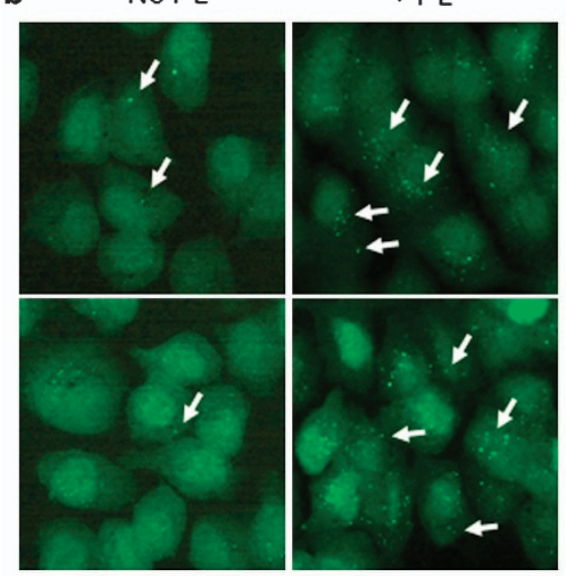

U2OS/GFP-LC3

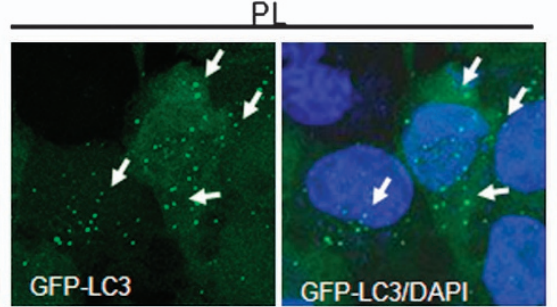

D NoPL

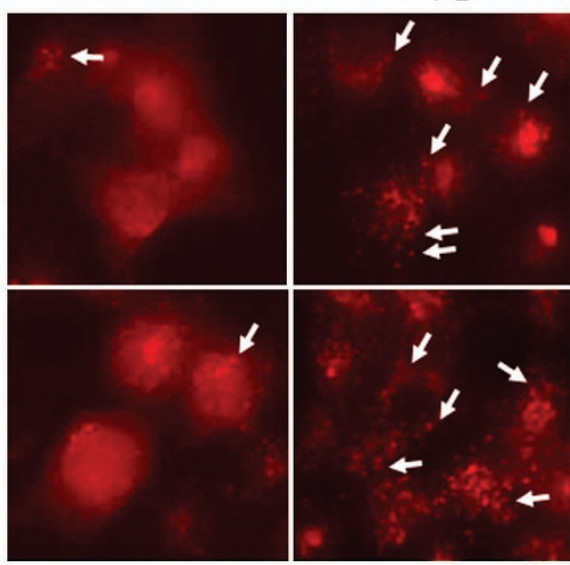

HeLa/mCherry-LC3 c

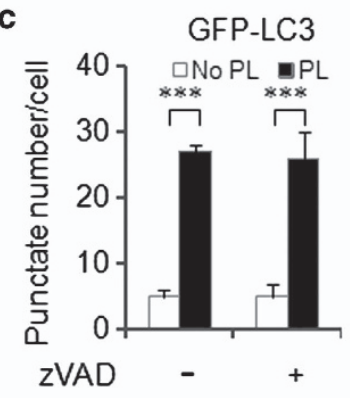

e

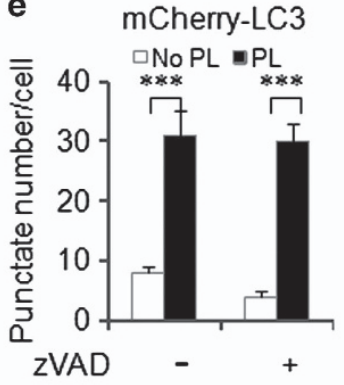

f

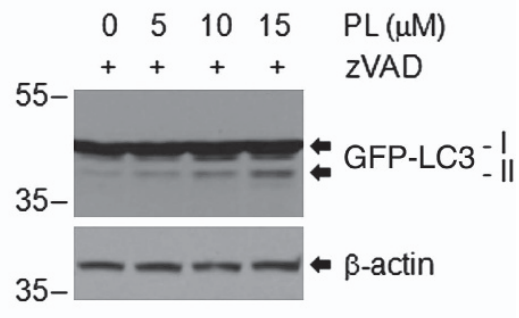

g
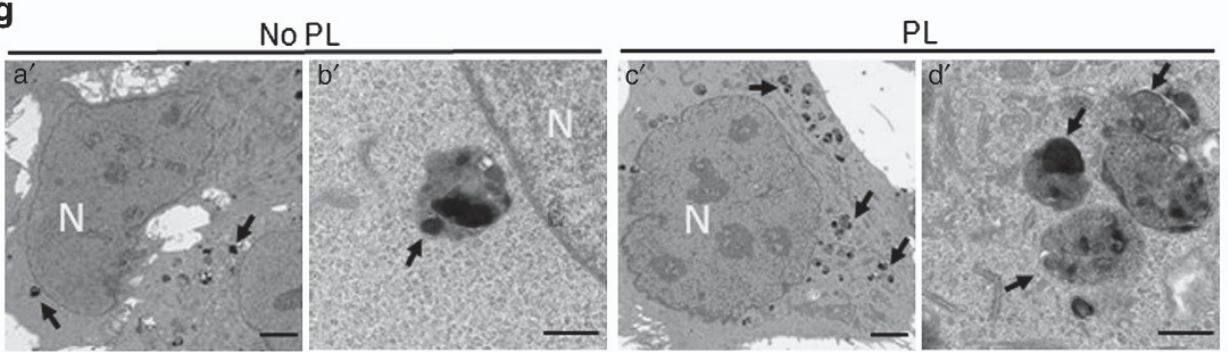

Figure 2 PL induces autophagy. (a) U2OS/GFP-LC3 cells were treated with PL $(2.5 \mu \mathrm{M})$ for $16 \mathrm{~h}$ before fixation with $4 \%$ paraformaldehyde. The cells were then stained with DAPI and analyzed with confocal microscopy. Arrows indicate the autolysosomes/autophagosomes. (b) U2OS/GFP-LC3 cells were pre-treated with zVAD (50 $\mu \mathrm{M})$ for $2 \mathrm{~h}$, followed by treatment with PL $(5 \mu \mathrm{M})$ for $16 \mathrm{~h}$. Punctate GFP-LC3 fluorescence (autolysosomes/autophagosomes) was indicated with white arrows. (c) The average punctate GFP-LC3 fluorescence dots were calculated in 200 cells. Data are shown as mean \pm S.D. (d and e) HeLa/mCherry-LC3 cells were pre-treated with zVAD ( $50 \mu M)$ for $2 \mathrm{~h}$, followed by treatment with PL $(5 \mu \mathrm{M})$ for $16 \mathrm{~h}$. (d) Punctate mCherry-LC3 fluorescence (autolysosomes/autophagosomes) was indicated with white arrows. (e) The average punctate mCherry-LC3 fluorescence dots were calculated in 200 cells. Data are shown as mean \pm S.D. ${ }^{* \star} P<0.001$. (f) Expression of GFP-LC3-I and -II in U2OS/GFP-LC3 stable cells treated with PL and zVAD as described in (b). Thirty micrograms of WCE proteins were loaded. GFP monoclonal antibody from Santa Cruz Biotechnology was used for the GFP-LC3 detection. GAPDH served as a loading control. (g) Electron micrograph of U2OS/GFP-LC3 cells treated with zVAD and PL for $24 \mathrm{~h}$. A large number of autolysosomes/autophagosomes were observed, but the nuclei were unaffected $\left(c^{\prime}\right)$. Black arrows indicated the autolysosomes/autophagosomes. The scale bars represent $20 \mu \mathrm{m}$ in $\mathrm{a}^{\prime}$ and $\mathrm{c}^{\prime}$ and $1 \mu \mathrm{m}$ in $\mathrm{b}^{\prime}$ and $\mathrm{d}^{\prime} . \mathrm{N}$, nucleus 
a

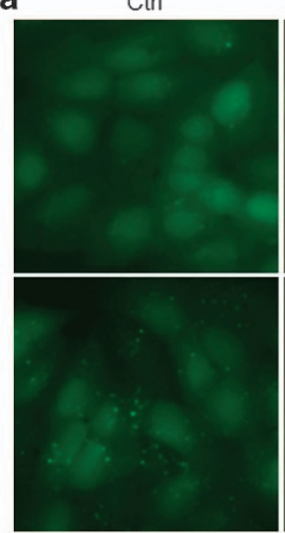

b

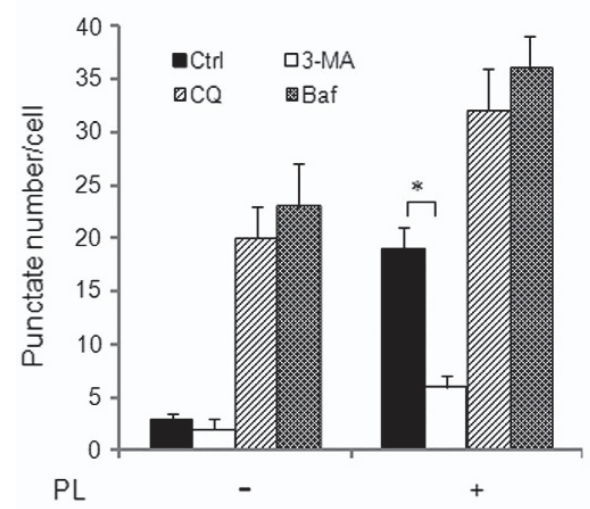

3-MA

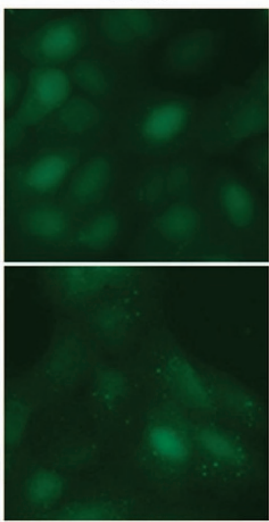

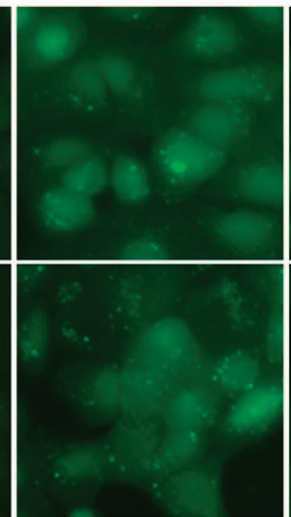
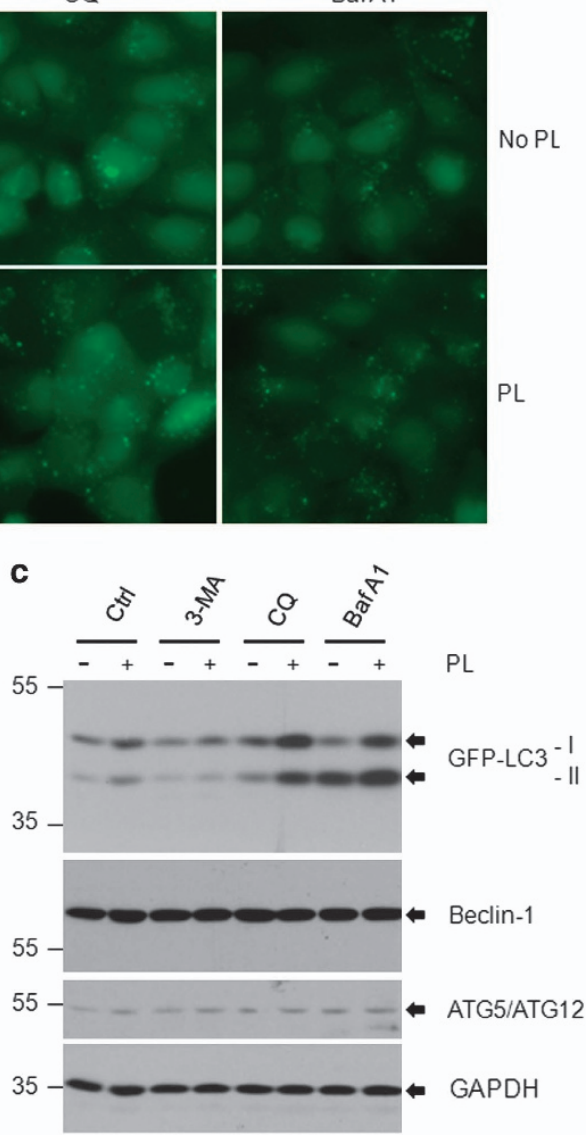

Figure $3 \mathrm{PL}$ enhances autophagy formation, does not block autophagy flux. U2OS/GFP-LC3 cells were pre-treated with $1 \mathrm{mM} 3-\mathrm{MA}, 10 \mu \mathrm{M} \mathrm{CQ}$, or $10 \mathrm{nM}$ Baf-A1 for $6 \mathrm{~h}$. zVAD $(50 \mu \mathrm{M})$ and PL $(5 \mu \mathrm{M})$ were then added. The cells were collected for the analysis post 16-h treatment. (a) Punctate GFP-LC3 fluorescence in U2OS/GFP-LC3 cells treated with PL in the presence or absence of various autophagy inhibitors. (b) The average numbers of punctate GFP-LC3 fluorescence dots were calculated in 200 cells. ${ }^{*} P<0.001$. (c) Expression of ATG5, Beclin-1, and GFP-LC3 in cells treated with autophagy inhibitors in ( $\mathbf{a}$ and $\left.\mathbf{b}\right)$ were determined with western blot. Thirty micrograms of WCE proteins were loaded. GAPDH served as a loading control

pre-treated the U2OS/GFP-LC3 cells with 3-methyladenine (3-MA), an autophagy inhibitor by blocking the class III PI3Ks that are critical during the late stage of vesicle expansion, followed by the addition of PL and z-VAD. PL-induced GFP-LC3 puncta were markedly reduced in the presence of 3-MA (Figures $3 a$ and $b$ ), suggesting that PL induces autophagy actively. To determine whether PL also affects the autophagy flux, we treated the cells with PL in the presence of two autophagy flux blockers, chloroquine (CQ) and bafilomycin A1 (Baf-A1). CQ prevents lysosome acidification and thereby the degradation of the products of autophagy, resulting in autophagolysosome accumulation, whereas Baf-A1 inhibits the maturation of autophagic vacuoles by preventing fusion between autophagosomes and lysosomes. As shown in Figures $3 a$ and $b$, co-treatment of $P L$ with $C Q$ or Baf-A1 markedly enhanced the accumulation of punctate GFP-LC3. Punctate numbers per cell were larger than that in cells treated with $\mathrm{PL}$ or $\mathrm{CQ} / \mathrm{Baf}-\mathrm{A} 1$ alone, suggesting that the action of PL on autophagy is not overlapped with $C Q$ and Baf-A1 and supporting the notion that $\mathrm{PL}$ enhances the formation of autophagy, thereby leading to further accumulation of autophagosomes/autolysosomes in the presence of autophagy flux blockers. Immunoblot also showed that LC3-II decreased markedly in PL-treated U2OS/GFP-LC3 cells in the presence of 3-MA and increased in the co-treatment with $\mathrm{CQ}$ or Baf-A1 as compared with cells treated with $\mathrm{PL}$ alone (Figure $3 \mathrm{c}$ ). Together, our results indicate that $\mathrm{PL}$ enhances autophagy formation, does not block autophagy flux.

Suppression of autophagy reduces PL-induced non-apoptotic cell death. To assess the role of autophagy in PL-induced non-apoptotic cell death, WT and ATG5deficient MEFs stably expressing mCherry-LC3 were treated with different concentrations of PL. PL treatment resulted in punctuate mCherry-LC3 in Atg5 $5^{+1+}$ mCherry-LC3 MEFs and much less punctuate mCherry-LC3 foci in Atg $5^{-1}$ mCherryLC3 MEFs (Supplementary Figure S2). Consistent with the resistance to autophagy induction, Atg5 $5^{-1-}$ MEFs were insensitive to $\mathrm{PL}$ treatment and maintained markedly higher levels of cell viability as compared with WT MEFs (Figures $4 \mathrm{a}$ and $\mathrm{b}$ ). Immunoblot verified LC3 lipidation (LC3-II) in WT cells and a lack of LC3-II signal in ATG5-null cells (Figure $4 \mathrm{c}$ ). To further validate the role of autophagy in 

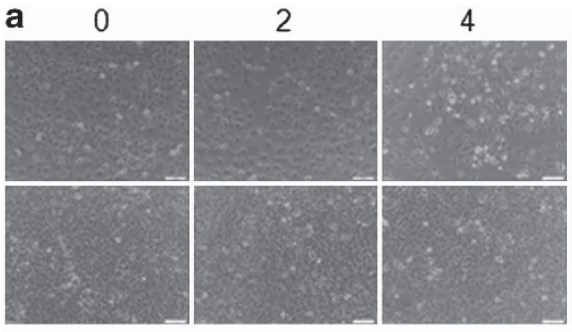

6
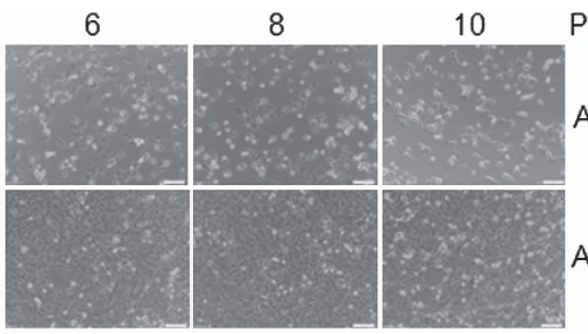

$\mathrm{PL}(\mu \mathrm{M})$

b

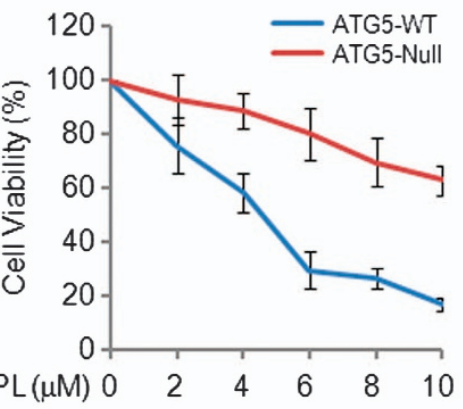

C

ATG5

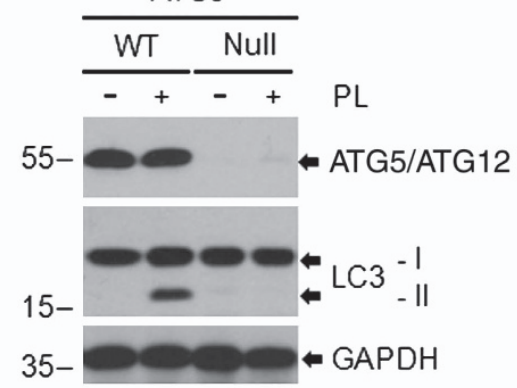

c

$$
\text { d }
$$

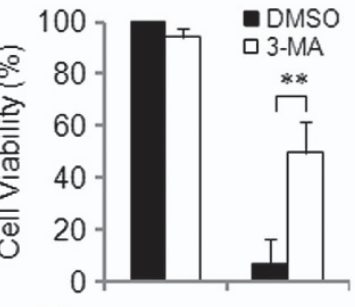

e

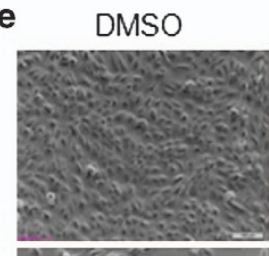

3-MA
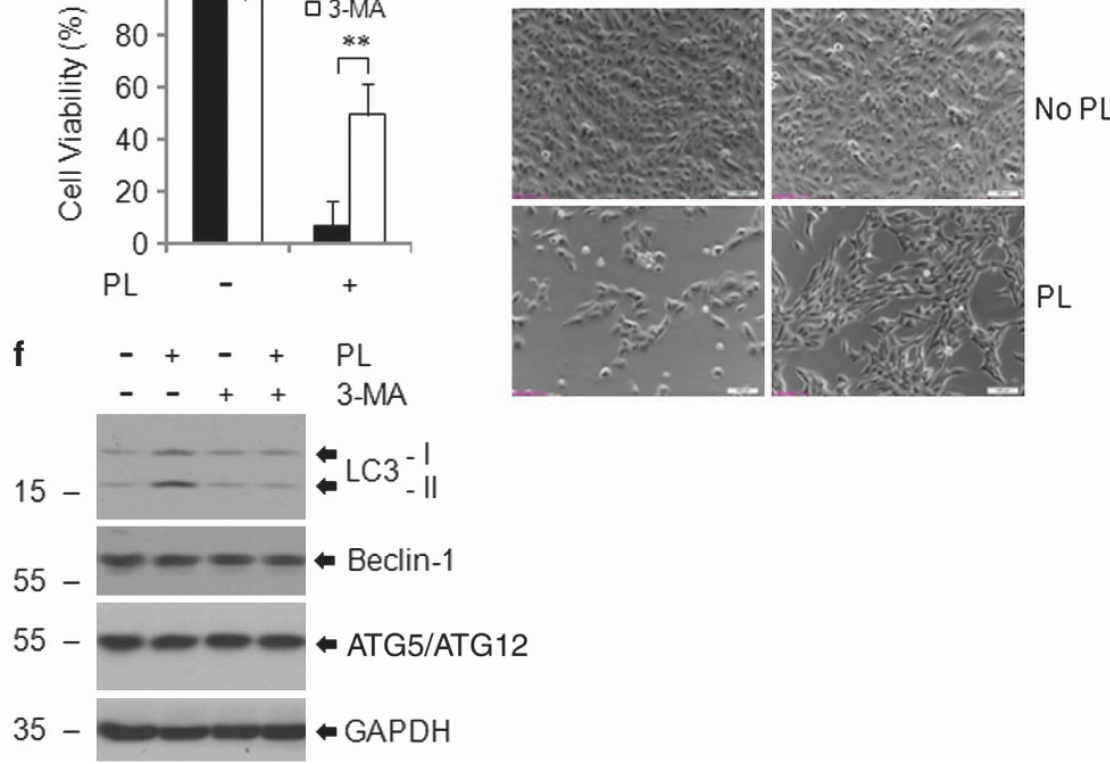

ATG5+/+

ATG5-/-

PL

Figure 4 PL-mediated autophagy is associated with cell death. (a-c) WT and null-ATG5 MEFs were exposed to $0-10 \mu M$ PL for $48 \mathrm{~h}$. (a) The cells were analyzed with phase contrast microscopy. (b) Cell viability was determined by MTT assay. Data are shown as mean \pm S.D. $(n=4)$. (c) Expression of ATG5 and LC3 in WT and null-ATG5 MEFs treated with PL in (a and $\mathbf{b})$ were determined with western blot. Thirty micrograms of WCE proteins were loaded. GAPDH served as a loading control. (d- $\mathbf{f})$ HeLa/ mCherry-LC3 cells were pre-treated with $1 \mathrm{mM} 3-\mathrm{MA}$ for $6 \mathrm{~h}$. The cells were then cultured in the presence or absence of PL (10 $\mu \mathrm{M})$ and zVAD $(50 \mu \mathrm{M})$ for $48 \mathrm{~h}$. (d) Cell viability was determined by MTT assay. Data are shown as mean \pm S.D. $(n=4) .{ }^{* \star} P<0.01$. (e) Cell morphology was analyzed with phase contrast microscopy. (f) Expression of LC3, ATG5 and Beclin-1 in cells treated with 3-MA in (d and $\mathbf{e}$ ) were determined with western blot. Thirty micrograms of WCE proteins were loaded. GAPDH served as a loading control

PL-induced non-apoptotic cell death, we blocked cell autophagy with 3-MA and assayed the cytotoxicity of PL on HeLa cells (Figures $4 d-f$ ). As shown previously, treatment with $\mathrm{PL}$ led to a considerable cell death although cell apoptosis was blocked by zVAD, verifying the existence of non-apoptotic cell death. More importantly, the cell death was substantially abrogated in the presence of 3-MA (Figures $4 \mathrm{~d}$ and $\mathrm{e}$ ). Thus, we conclude that autophagy contributes to PL-induced non-apoptotic cell death.
PL-induced autophagy is not due to disruption of cellular bioenergetics homeostasis. To gain an insight into the mechanism by which PL induces autophagy, we sought to determine whether disruption of cellular bioenergetics is involved in this process. As shown in Figure $5 \mathrm{a}$, cellular ATP levels were not altered by the treatment of $\mathrm{PL}$ in U2OS/GFP-LC3 cells. Methyl pyruvate (MP), a small molecule that can permeablize into the cells, oxidized in the mitochondria for ATP synthesis, could not block the 


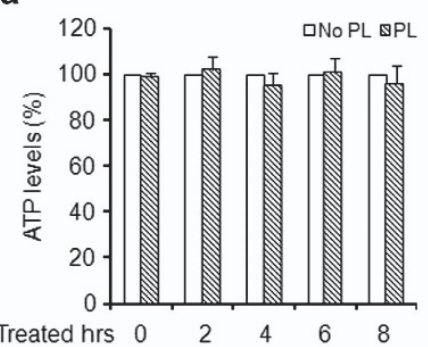

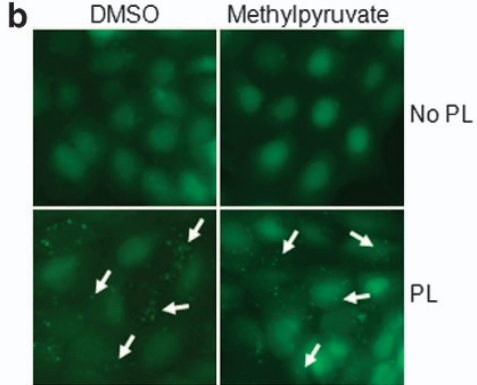

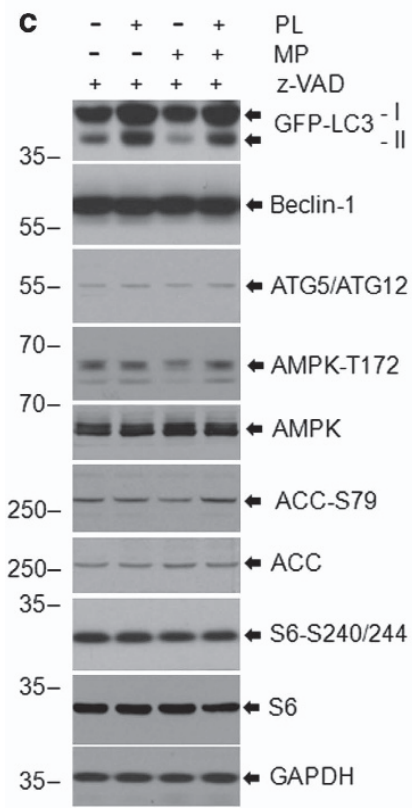

d DMSO NAC
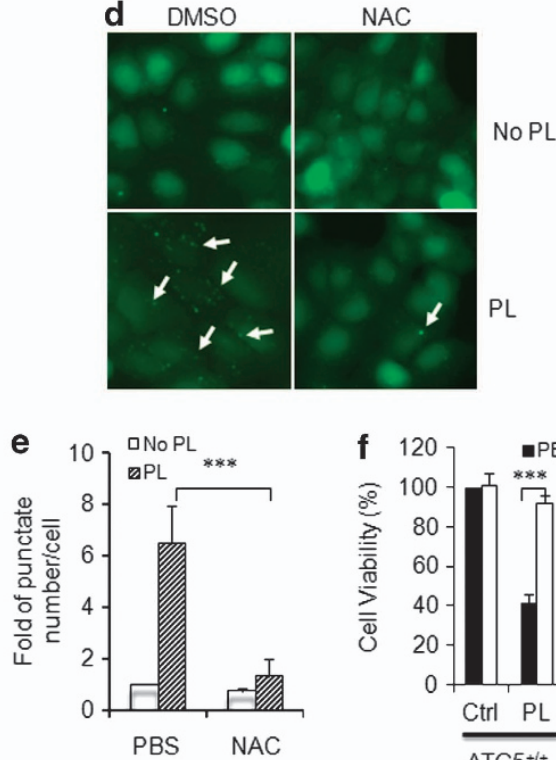

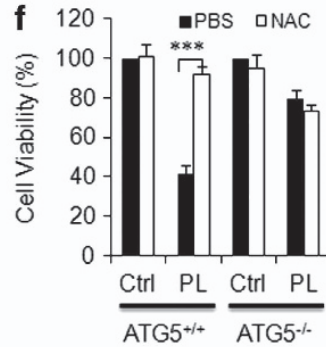

Figure $5 \mathrm{PL}$ induces autophagy by increasing intracellular ROS. (a) U2OS/GFP-LC3 cells were treated with $10 \mu \mathrm{M} \mathrm{PL}$ for 0-8 h. Cell lysates were collected for the determination of ATP levels with a Bioluminescence Assay Kit CLS II from Roche Scientific (Indianapolis, IN, USA). (b) U2OS/GFP-LC3 cells were pre-treated with 1 mM MP for $6 \mathrm{~h}$, followed by treatment with PL $(10 \mu \mathrm{M})$ for $16 \mathrm{~h}$. GFP-LC3 fluorescence was captured with fluorescence microscopy. White arrows indicate punctate GFP-LC3 fluorescence (autolysosomes/autophagosomes). (c) Expression of autophagy regulative genes and AMPK signaling molecules in U2OS/GFP-LC3 cells treated with PL and/or MP by western blot. Thirty micrograms of WCE proteins were loaded. GAPDH served as a loading control. (d and e) U2OS/GFP-LC3 cells were pre-treated with 5 mM NAC for $6 \mathrm{~h}$, followed by treatment with PL $(10 \mu \mathrm{M})$ for $16 \mathrm{~h}$. GFP-LC3 fluorescence was captured with fluorescence microscopy. White arrows indicate punctate GFP-LC3 fluorescence (autolysosomes/autophagosomes) in (d). Folds of punctate GFP-LC3 numbers are shown in (e). (f) WT and null-ATG5 MEFs were pre-treated with 5 mM NAC for 6 h, followed by treatment with $10 \mu \mathrm{MPL}$ for $48 \mathrm{~h}$. Cell viability was determined by MTT assay. Data are shown as mean \pm S.D. $(n=4) .{ }^{* * *} P<0.001$

formation of punctuate fluorescent vesicles and LC3 lipidation in PL-treated cells (Figures $5 \mathrm{~b}$ and $\mathrm{c}$ ), suggesting that PL-associated autophagy induction is independent of cellular bioenergetic stress. AMP-activated protein kinase (AMPK), a cellular energy sensor, responds to cellular energy deficiency or pseudo starvation and triggers autophagy through the mTOR cascade. To determine whether $P L$ exerts a pseudo starvation effect to induce AMPK activation, we exposed the WT and AMPK $\alpha$-null MEFs to different concentrations of PL. As shown in Supplementary Figure S3, cell viability was only marginally decreased in AMPK $\alpha$-null MEFs treated with PL as compared with WT MEFs, suggesting that AMPK is not associated with cellular sensitivity to PL. Consistent with this finding, immunoblot showed that $\mathrm{PL}$ treatment did not alter the levels of pAMPK-T172, pACC-S79 and pS6-S240/244, suggesting that the AMPK-TSC2-mTORC1 cascade is not affected by PL (Figure 5c). Also, addition of MP had little effect on PL action (Figure 5c). Thus, we conclude that PL-induced autophagy is not due to disruption of cellular bioenergetics.

PL-induced elevation of ROS contributes to autophagy. Previous reports showed that PL elevates intracellular ROS and selectively kills cancer cells. ${ }^{28,34}$ ROS are recognized as an inducer of autophagy. To validate the induction of ROS by $\mathrm{PL}$ and to determine the role of ROS in PL-mediated autophagy, we first detected the ROS levels in PL-treated U2OS, MEF and HeLa cells through flow cytometry using the redox-sensitive fluorescent probe dichlorofluorescein diacetate. Indeed, treatment with $\mathrm{PL}$ for $4 \mathrm{~h}$ led to 6- to 11-fold increases of the ROS levels in the cells (Supplementary Figure S4). Pre-treatment with $5 \mathrm{mM}$ $\mathrm{N}$-acetyl-cysteine (NAC), an ROS scavenger, prevented the ROS induction by the PL (Supplementary Figure S4). Consistently, PL-induced autophagy was also abrogated by 
a
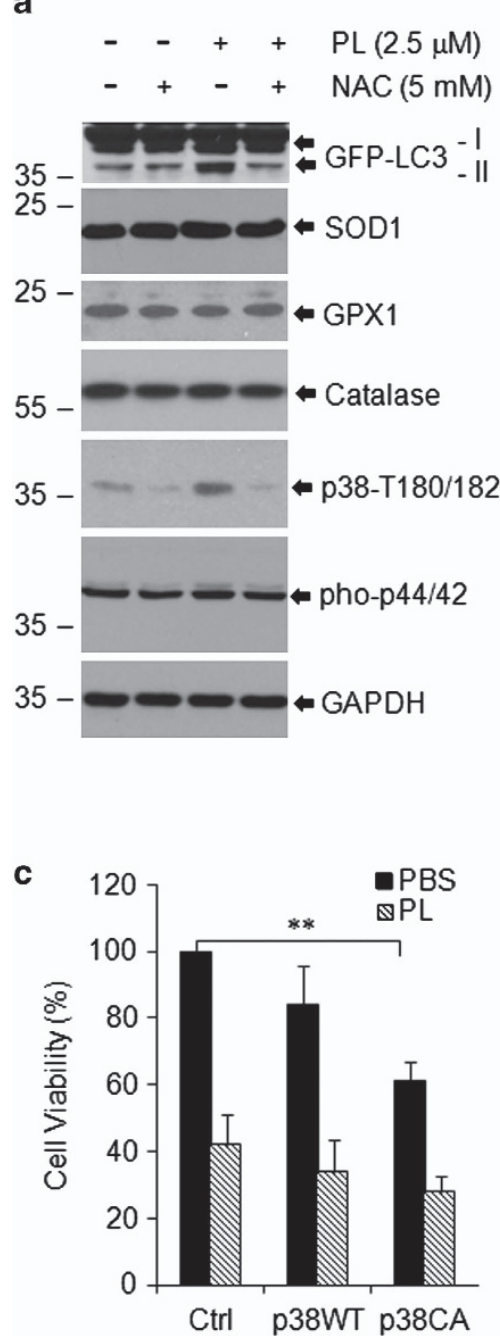

b
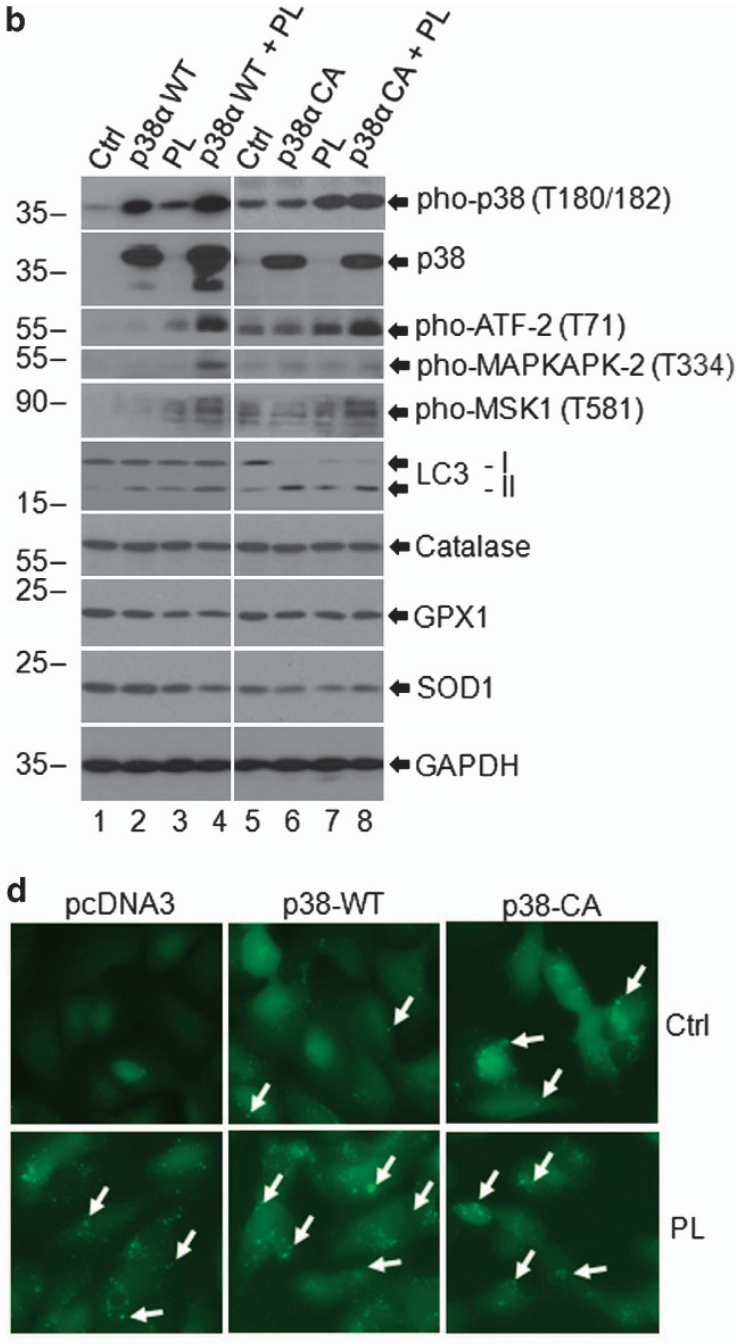

Figure 6 PL activates p38 signaling. (a) U2OS/GFP-LC3 cells were pre-treated with $5 \mathrm{mM} \mathrm{NAC}$ for $6 \mathrm{~h}$, followed by treatment with $2.5 \mu \mathrm{M}$ PL for $16 \mathrm{~h}$. The WCE was isolated after the treatment. Thirty micrograms of total proteins were loaded for western blot analysis. GAPDH served as a loading control. (b and $\mathbf{c}$ ) U2OS cells were transfected with pcDNA3 (Ctrl), pcDNA3/p38-WT and pcDNA3/p38-CA and selected with $200 \mu \mathrm{g} / \mathrm{ml}$ hygromycin for 2 weeks for the establishment of stable expression cell lines. (b) The control and p38 stable expression cells were treated with $10 \mu \mathrm{M} \mathrm{PL}$ for $16 \mathrm{~h}$. After the treatment, the WCE was isolated for western blot detection of p38 signaling targets, anti-oxidant enzymes, and autophagy marker (LC3). Thirty micrograms of total proteins were loaded. GAPDH served as a loading control. (c) The control and p38 stable expression cells were treated with $10 \mu \mathrm{M} \mathrm{PL}$ for $48 \mathrm{~h}$. The cell viability was determined with MTT assay after the treatment. Data are shown as mean \pm S.D. $(n=4)$. ${ }^{\star \star} P<0.01$. (d) U2OS/GFP-LC3 cells were transfected with pcDNA3 (Ctrl), pcDNA3/p38-WT or pcDNA3/p38-CA for $48 \mathrm{~h}$. The cells were then exposed to $10 \mu \mathrm{M}$ PL for $16 \mathrm{~h}$. GFP-LC3 fluorescence was captured with fluorescence microscopy. White arrows indicate punctate GFP-LC3 fluorescence (autolysosomes/autophagosomes)

co-treatment with NAC (Figures $5 \mathrm{~d}$ and e), indicating that ROS mediated the autophagy formation in PL-treated cells.

To determine the role of ROS in PL-mediated nonapoptotic cell death, U2OS/GFP-LC3 cells and HeLa cells were pre-treated with $\mathrm{ZVAD}$ followed by co-treatment of NAC and PL. Cell viability was maintained at substantially higher levels in the presence of NAC (Supplementary Figure S5 and data not shown), indicating that ROS are indeed involved in PL-induced non-apoptotic cell death. To further determine the role of autophagy in the non-apoptotic cell death triggered by PL and mediated through ROS elevation, we detected the cytotoxicity of PL to WT and ATG5 null MEFs in the presence or absence of NAC. Treatment of $\mathrm{PL}$ resulted in decreased cell viability in autophagy intact WT cells. Co-treatment with NAC markedly enhanced the viability of PL-treated cells (Figure $5 f$ ). In contrast, autophagy-deficient ATG5-null MEFs showed marginal cell viability alteration irrespective of the presence or absence of PL/NAC (Figure 5f). Taken together, these data suggest that PL-induced elevation of ROS contributes to autophagyassociated non-apoptotic cell death.

PL activates p38 signaling. p38 and its mediated signaling are critical targets responding to ROS stress. ${ }^{35-37}$ Thus, we questioned whether PL-induced autophagy mediated by ROS is associated with the activation of p38 signaling. As shown in Figure 6a and Supplementary Figure S6, phosphorylation of p38 (T180/182) was indeed increased with PL treatment, which could be blocked with NAC, whereas phospho-p44/42, another member of the mitogen-activated 
a

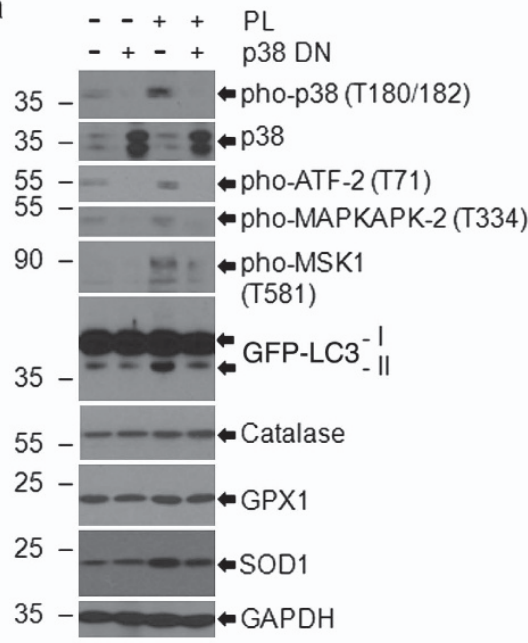

d

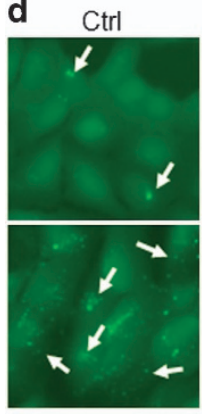

SB203580

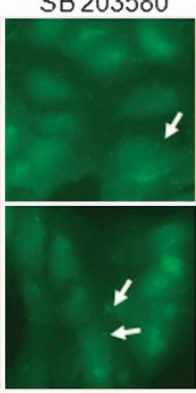

e

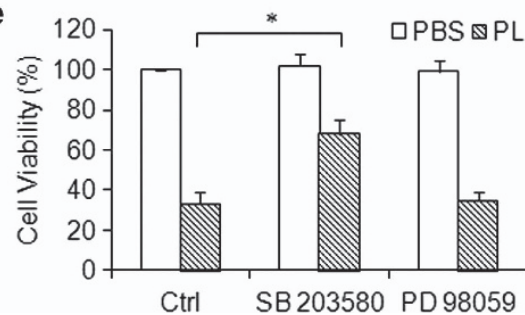

PD 98059 b

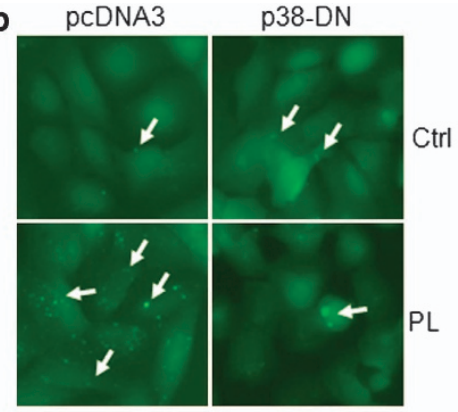

C
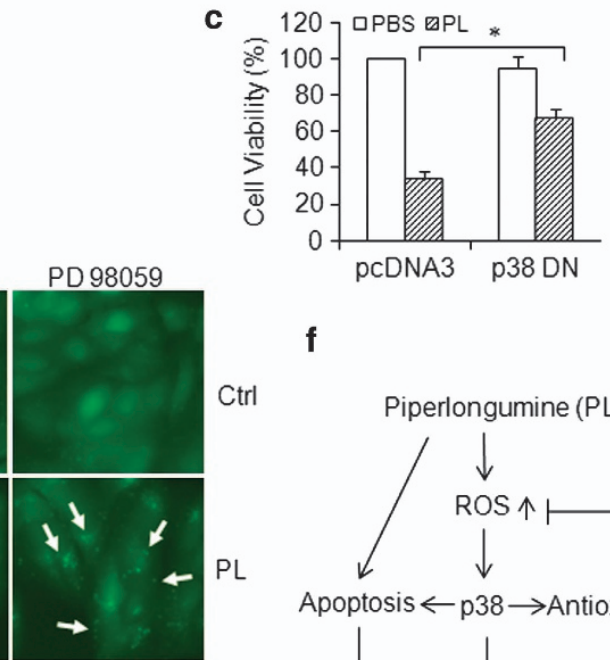

f

Ctrl

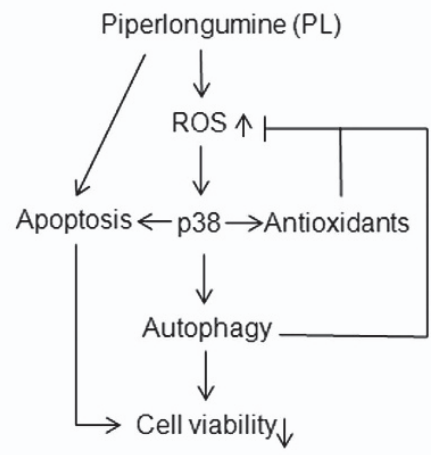

Figure 7 p38 signaling is required for autophagy induction by PL. (a-c) U2OS/GFP-LC3 cells were transfected with pcDNA3 (Ctrl) or pcDNA3/p38-DN constructs and selected with $200 \mu \mathrm{g} / \mathrm{ml}$ hygromycin for 2 weeks for the establishment of stable expression cell lines. (a) The control and p38-DN stable expression cells were treated with $10 \mu \mathrm{M} \mathrm{PL}$ for $16 \mathrm{~h}$. After the treatment, the WCE was isolated for western blot detection of p38 signaling targets, anti-oxidant enzymes and autophagy marker (LC3). Thirty micrograms of total proteins were loaded. GAPDH served as a loading control. (b) The control and p38-DN stable expression cells were treated with $10 \mu \mathrm{M}$ PL for $16 \mathrm{~h}$. GFP-LC3 fluorescence was captured with fluorescence microscopy after the treatment. White arrows indicate punctate GFP-LC3 fluorescence (autolysosomes/ autophagosomes). (c) The control and p38-DN stable expression cells were treated with $10 \mu \mathrm{M} \mathrm{PL}$ for $48 \mathrm{~h}$. The cell viability was determined with MTT assay after the treatment. Data are shown as mean \pm S.D. $(n=4)$. ${ }^{\star} P<0.05$. (d) U2OS/GFP-LC3 cells were pre-treated with $10 \mu \mathrm{M} \mathrm{SB} 203580$, a p38 inhibitor, or $20 \mu \mathrm{M}$ PD 98059 , a p44/ 42 inhibitor for $6 \mathrm{~h}$, followed by treatment with $10 \mu \mathrm{M} \mathrm{PL}$ for $16 \mathrm{~h}$. GFP-LC3 fluorescence was captured with fluorescence microscopy after the treatment. White arrows indicate punctate GFP-LC3 fluorescence (autolysosomes/autophagosomes). (e) U2OS/GFP-LC3 cells were pre-treated with $10 \mu \mathrm{M} \mathrm{SB} 203580$, a p38 inhibitor or $20 \mu \mathrm{M}$ PD 98059 , a p44/42 inhibitor for $6 \mathrm{~h}$, followed by treatment with $10 \mu \mathrm{M}$ PL for $48 \mathrm{~h}$. The cell viability was determined with MTT assay after the treatments. Data are shown as mean \pm S.D. $(n=4)$. ${ }^{*} P<0.05$. (f) Role of $\mathrm{PL}$ in autophagy. Schematic diagram depicts mechanism of action of PL to induce autophagy. PL increases intracellular ROS levels, thereby activating p38 signaling and enhancing cell autophagy

protein kinase (MAPK) family exhibited no alteration, suggesting that PL specifically induces activation of the p38 pathway. PL treatment only marginally reduced antioxidant enzyme, glutathione peroxidase 1 (GPX1) expression, and had no effect on SOD and catalase levels in U2OS/GFP-LC3 cells either in the presence or absence of NAC.

To further validate the involvement of p38 signaling in PL-induced autophagy, U2OS cells were transfected with pcDNA3 (Ctrl), pcDNA3/p38-WT and pcDNA3/constitutively active p38 (p38-CA) and selected with hygromycin for the establishment of stable cell lines. As shown in Figure $6 \mathrm{~b}$, expression of total p38 markedly increased in p38-WT and p38-CA transfected U2OS cells as compared with control cells. p38 signaling downstream targets, such as phosphorylation of activating transcription factor 2 (ATF-2), MAP kinase-activated protein kinase 2 (MAPKAPK-2) and mitogen- and stress-activated protein kinase 1 (MSK1), were upregulated in p38CA and to a less level in p38-WT stable 
cells. PL treatment substantially elevated the phosphorylation of p38 and activated the downstream targets of the signaling. Phosphorylation of ATF-2 and MSK1 was further increased in p38-WT transfected cells treated with PL (lane 4, Figure 6b) as compared with untreated p38-WT stable cells (lane 2, Figure $6 \mathrm{~b}$ ) or PL-treated control cells (lane 3, Figure 6b). PL treatment exhibited only a marginal additional effect to the activation of p38 downstream signaling in p38-CA cells as compared with PL-treated control cells (compare lane 8 with lane 7, Figure 6b). Moreover, cell viability was markedly reduced in p38-CA or p38-WT transfected cells as compared with control cells even in the absence of $\mathrm{PL}$ treatment (Figure 6c). In addition, LC-II levels were increased in p38-CA transfected U2OS cells as revealed by western blot (Figure 6b). In consistent with these findings, p38-CA transfected U2OS/GFP-LC3 cells showed markedly increased GFP-LC3 puncta and PL treatment had no further effect (Figure 6d). Taken together, these data suggest that PL treatment activates p38 signaling, which may be associated with enhanced autophagy activity.

p38 signaling is required for autophagy induction by PL. To further elucidate the role of p38 activation in PL-induced autophagy, expression of a dominant-negative p38 construct (p38-DN) and a specific p38 kinase inhibitor were used to inhibit p38 signaling. As shown in Figure 7a, expression of total p38 was markedly increased in p38-DN transfected U2OS/GFP-LC3 cells, whereas phosphorylation of p38 decreased markedly in the cells irrespective of the presence or absence of PL. Accordingly, p38 signaling downstream targets, such as phosphorylation of ATF-2, MAPKAPK-2 and MSK1, were inhibited in the p38-DN cells treated with or without PL (Figure 7a). Autophagy marker LC-II decreased significantly in p38-DN transfected cells as compared with control cell upon the treatment of PL (Figure 7a), which is consistent with reduced autophagosome formation as revealed by punctate GFP-LC3 fluorescence (Figure 7b). Furthermore, cell viability was markedly improved in p38-DN cells treated with PL as compared with control cells (Figure 7c). These results suggest that the integrity of p38 signaling is necessary for PL-induced autophagy and its associated cell death.

Next, we blocked p38 signaling with a specific p38 kinase inhibitor, SB 203580. SB 203580 is a pyridinyl imidazole that suppresses the activation of $\mathrm{p} 38 \alpha$ and $\mathrm{p} 38 \beta$, thereby reducing the activation of downstream targets, such as MAPKAPK-2 and heat shock protein 27. Application of SB 203580 substantially reduced the autophagosome formation in PLtreated cells (Figure 7d) and improved cell viability upon PL treatment (Figure 7e). In stark contrast, PD98059 (2'-amino$3^{\prime}$-methoxyflavone), a flavone derivative and selective p44/42 MAPK kinase (MEK1/MEK2) inhibitor, did not affect PL-associated autophagosome formation and cell viability (Figures $7 d$ and e). Taken together, our results suggest that p38 signaling is required for PL-induced autophagy and autophagy-associated cell death.

\section{Discussion}

In this study, we showed that PL induces tumor cell death in the presence of zVAD-fmk and Nec-1, suggesting the existence of non-apoptotic and non-necrotic cell death. We further demonstrated that this cell death is mediated by autophagy, which can be suppressed by 3-MA, an autophagy inhibitor, and be substantially reduced in Atg5-deficient cells. We verified that PL enhances autophagy formation, but does not block autophagy flux. In addition, we characterized that PL activates the p38 signaling and induces p38-dependent stress response and autophagy formation. Thus, our results suggest that PL not only induces apoptosis, but also triggers nonapoptotic and non-necrotic cell death, which is associated with autophagy induction.

Autophagy maintains cellular homeostasis in normal tissue. However, the role of autophagy in cancer is multifaceted, which can either suppress or support the tumor growth depending on the cellular context. ${ }^{1-5}$ The induction of autophagy may allow the stressed cells to survive in the tumor microenvironment. However, if levels of autophagy are induced beyond a physiological range, the autophagy pathway can contribute to cell death. Consistent with this idea, many cancer cells might have lost the ability to undergo this form of non-apoptotic death as a growth advantage. ${ }^{14}$ On the other hand, induction of autophagy has been tested for a potential therapeutic strategy of cancer. Chen et al. ${ }^{38}$ reported that rotenone and thenoyltrifluoroacetone (TTFA) induce ROS, and the ROS scavenger tiron decreased autophagy and cell death induced by rotenone and TTFA in HeLa cells. By contrast, treatment of nontransformed primary mouse astrocytes with rotenone or TTFA failed to increase ROS or autophagy, suggesting that through a ROS-mediated mechanism, cancer cells could be selectively targeted by autophagic cell death. For another example, Singh's group found that phenethyl isothiocyanate, a bioactive constituent of many edible cruciferous vegetables, suppressed prostate cancer progression by inducing ROS-related autophagic cell death. ${ }^{39,40}$ In this study, we demonstrated that PL-induced autophagy is due to elevated intracellular ROS. Application of NAC, an antioxidant, markedly reduces PL-induced autophagy formation and cell death. Thus, together with previous reports showing that PL could selectively kill cancer cells through the ROS mechanism, ${ }^{27-29}$ our results add additional support to the generable application of ROS inducers by triggering autophagy-associated cell death.

The p38 MAPK is an important stress kinase that is involved in inflammation, cell growth and differentiation, cell cycle, and cell death. Recent report showed that the ability of p38 MAPK to induce apoptosis in response to ROS has an inhibitory role in tumor initiation. ${ }^{35}$ In this report, we found that PL activates p38 signaling, whose responses overlapped with constitutive active form of p38. In contrast, dominant-negative p38 and p38 inhibitor abrogated PL-induced autophagy and cell death, suggesting that p38 signaling is indispensable for the action of PL. In addition, p44/42 MAPK could not be phosphorylated by $\mathrm{PL}$ treatment, further suggesting the specificity of the p38 response. We did not observe the activation of p38 upstream MAP kinase kinases (MKKs) MKK3, MKK6 or MKK4. It has been shown that these and several other kinases can phosphorylate p38 under stress conditions. Therefore, we postulate that these upstream kinases may be regulated by PL-induced ROS. The p38 MAPK substrates ATF-2 and MSK1 are phosphorylated after the treatment of PL (Figures $6 b$ and 7a). As a transcription 
factor, ATF-2 may drive the expression of genes implicated in autophagy induction. Taken together, our results suggest that p38 not only facilitates the stress response to PL but also has an essential role in PL-induced cell death (Figure 7f).

There exist conflicting data about the role of p38 MAPK in autophagy. Depending on the stimulus, it can be considered as either an inducer or an inhibitor of autophagy. ${ }^{41-45}$ For example, Zhan et al. ${ }^{41}$ recently reported that MS275, a synthetic benzamide derivative of the histone deacetylase inhibitor, induces autophagy as well as apoptosis in human colon cancer cells. The autophagy induction is dependent on intracellular ROS production and p38 signaling activation. For another example, Moruno-Manchon et al. ${ }^{42}$ have recently identified p38 MAPK as an important effector in the induction of autophagy by glucose. In contrast, other researchers reported that p38 inhibition can actually lead to autophagy. de la Cruz-Morcillo et al. ${ }^{43,44}$ showed that p38 MAPK is a major determinant of the balance between apoptosis and autophagy under the treatment of genotoxic drugs. In the same way, inhibition of p38 MAPK has been shown to induce autophagy by increasing the expression of Beclin-1 and LC3. Keil et al. ${ }^{45}$ recently showed that the growth arrest and DNA damage 45 beta-MAPK/ERK kinase kinase 4 pathway specifically directs p38 to autophagosomes. This process results in an accumulation of autophagosomes through p38-mediated inhibition of lysosome fusion. It was further demonstrated that Atg5 is phosphorylated by p38 at threonine 75, which is responsible for the inhibition of starvation-induced autophagy. It is worthy to mention that many of these studies were based on the use of the chemical inhibitors SB203580 or SB202190, which may produce off-target effects on other proteins such as $\mathrm{PI}$ IK/Akt or AMPK. Therefore, it is necessary in the future to further clarify the role of p38 MAPK in autophagy and assess the implication of p38-associated autophagy in chemo- and radiation therapy.

Taken together, our results clarify that PL can induce autophagic cell death by activation of p38 in the presence of pan-caspase inhibitor, indicating the therapeutic potential of $\mathrm{PL}$ in apoptosis-resistant cancers. If results from this study warrant in the in vivo investigations in the future, we anticipate that oxidative stress inducers, such as PL, can be an effective means of selectively eradicating cancer cells, which sustain high levels of ROS and are more dependent on anti-oxidant for the survival and susceptible to oxidative stress inducers. As PL is a natural compound found in vegetables with low toxicity to normal cells, its applications for clinical treatment of cancers are feasible and highly significant.

\footnotetext{
Materials and Methods

Antibodies and chemicals. Antibody against caspase-7 was purchased from BD Pharmingen (San Diego, CA, USA). Antibodies against S6, S6-S240/244, AMPK, AMPK-T172, ACC and ACC-S79, p38-T180/182, pho-p44/42 (Thr 202/Tyr 204), p38, pho-ATF-2 (T71), pho-MAPKAPK-2 (T334), and pho-MSK1 (T581) were purchased from Cell Signaling (Beverly, MA, USA). Anti- human RIP1, RIP3, SOD1, GPX1, catalase and PGAM5 antibodies were from Abcam Inc. (Cambridge, MA, USA). Anti-Atg5 antibody was purchased from ProteinTech Group Inc. (Chicago, IL, USA). Antibodies against Beclin-1 and LC3 were chased from Novus Biological Inc. (Littleton, CO, USA). Anti-GFP monoclonal antibody was purchased from Santa Cruz Biotechnology (Santa Cruz, CA, USA). PL, Nec-1, 3-MA, CQ, Baf-A1, MP, and NAC, SB 203580 (p38 inhibitor), and PD 98059 (p44/42 inhibitor) were purchased from Sigma-Aldrich (St Louis, MO,
}

USA). zVAD-fmk was purchased from Biomol International (Plymouth Meeting, PA, USA).

Cell lines and DNA transfection. U2OS, HeLa cells, WT, and ATG5 $5^{-1-}$ or $\mathrm{AMPK}^{-/-}$MEFs were grown in Dulbecco's modified Eagle's medium containing $10 \%$ fetal bovine serum in a humidified incubator containing $5 \% \mathrm{CO}_{2}$ at $37{ }^{\circ} \mathrm{C}$. The establishments of U2OS/GFP-LC3 and HeLa/mCherry-LC3 cells were reported previously. ${ }^{26}$ U2OS cells were transfected with pcDNA3 (Ctrl), pcDNA3/p38-WT, pcDNA3/p38-CA or pcDNA3/p38-DN and selected with $200 \mu \mathrm{g} / \mathrm{ml}$ hygromycin for 2 weeks for the establishment of stable expression cell lines.

Immunofluorescence and fluorescence microscopy. The cells were grown in six-well plates with cover slides and fixed in cold $4 \%$ neutral paraformaldehyde in PBS for 30 min on ice, washed in PBS, permeabilized with $1 \%$ Triton X-100, 0.5\% NP-40 in PBS and blocked in $1 \%$ bovine serum albumin in PBS. Incubation with a primary antibody was carried out for $2 \mathrm{~h}$ at room temperature. Incubation with a secondary antibody was carried out for $1 \mathrm{~h}$ at room temperature. Slides were mounted with Vectashield antifade medium (Vector Laboratories, Burlingame, CA, USA) containing 4,6-diamidino-2-phenylindole (DAPI) after three washes with washing buffer and examined under fluorescence microscope. The location and distribution of GFP-LC3 staining were examined directly as described previously using fluorescence microscope. ${ }^{26}$

Immunoblotting. Cells were collected in RIPA lysis buffer. Immunoblotting was performed as described previously. ${ }^{26}$ A total of $30 \mu \mathrm{g}$ protein was used for the immunoblotting, unless otherwise indicated. GAPDH or $\beta$-actin was used for the loading control.

Cell viability and cell death assay. Cell viability was measured by the MTT assay as described previously. ${ }^{46}$ Cell death was determined by Trypan blue (Sigma-Aldrich) exclusion assay.

Statistical analysis. The unpaired $t$-test was used for the statistical analyses between two groups. $P<0.05$ was considered statistically significant.

\section{Conflict of Interest}

The authors declare no conflict of interest.

Acknowledgements. This work was supported by grants from National Cancer Institute R01CA133053, the Cervical Cancer SPORE Career Development Award and Pilot Award from NCI P50CA098252, and the Biomedical Research Foundation (ZXX), the National 863 Program \#2004AA205020 and the National Natural Science Foundation of China \#30700872 (YLL).

1. Mizushima N. Autophagy: process and function. Genes Dev 2007; 21: 2861-2873.

2. Kimmelman AC. The dynamic nature of autophagy in cancer. Genes Dev 2011; 25 1999-2010.

3. Choi AM, Ryter SW, Levine B. Autophagy in human health and disease. $N$ Engl J Med 2013; 368: 651-662.

4. Levine B. Cell biology: autophagy and cancer. Nature 2007; 446: 745-747.

5. Levine B, Kroemer G. Autophagy in the pathogenesis of disease. Cell 2008; 132: 27-42.

6. Levine B, Mizushima N, Virgin HW. Autophagy in immunity and inflammation. Nature 2011; 469: 323-335.

7. Cadwell K, Liu JY, Brown SL, Miyoshi H, Loh J, Lennerz JK et al. A key role for autophagy and the autophagy gene Atg16l1 in mouse and human intestinal Paneth cells. Nature 2008; 456: 259-263.

8. Lee HK, Mattei LM, Steinberg BE, Alberts $\mathrm{P}$, Lee YH, Chervonsky A et al. In vivo requirement for Atg5 in antigen presentation by dendritic cells. Immunity 2010; 32: 227-239.

9. Lum JJ, Bauer DE, Kong M, Harris MH, Li C, Lindsten T et al. Growth factor regulation of autophagy and cell survival in the absence of apoptosis. Cell 2005; 120: 237-248.

10. Degenhardt K, Mathew R, Beaudoin B, Bray K, Anderson D, Chen G et al. Autophagy promotes tumor cell survival and restricts necrosis, inflammation, and tumorigenesis. Cancer Cell 2006; 10: 51-64.

11. Amaravadi RK, Yu D, Lum JJ, Bui T, Christophorou MA, Evan Gl et al. Autophagy inhibition enhances therapy-induced apoptosis in a Myc-induced model of lymphoma. J Clin Invest 2007; 117: 326-336. 
12. Karantza-Wadsworth V, Patel S, Kravchuk O, Chen G, Mathew R, Jin S et al. Autophagy mitigates metabolic stress and genome damage in mammary tumorigenesis. Genes Dev 2007; 21: 1621-1635.

13. Mathew R, Kongara S, Beaudoin B, Karp CM, Bray K, Degenhardt K et al. Autophagy suppresses tumor progression by limiting chromosomal instability. Genes Dev 2007; 21 1367-1381

14. Hippert MM, O'Toole PS, Thorburn A. Autophagy in cancer: good, bad, or both? Cancer Res 2006; 66: 9349-9351.

15. Jin S, White E. Role of autophagy in cancer: management of metabolic stress. Autophagy 2007; 3: 28-31.

16. Liang C, Feng P, Ku B, Dotan I, Canaani D, Oh BH et al. Autophagic and tumour suppressor activity of a novel Beclin1-binding protein UVRAG. Nat Cell Biol 2006; 8 688-699.

17. Takamura A, Komatsu M, Hara T, Sakamoto A, Kishi C, Waguri S et al. Autophagydeficient mice develop multiple liver tumors. Genes Dev 2011; 25: 795-800.

18. Inami $Y$, Waguri S, Sakamoto A, Kouno T, Nakada K, Hino O et al. Persistent activation of Nrf2 through p62 in hepatocellular carcinoma cells. J Cell Biol 2011; 193: 275-284.

19. Guertin DA, Sabatini DM. Defining the role of mTOR in cancer. Cancer Cell 2007; 12: 9-22.

20. Diaz-Troya S, Perez-Perez ME, Florencio FJ, Crespo JL. The role of TOR in autophagy regulation from yeast to plants and mammals. Autophagy 2008; 4: 851-865.

21. Maiuri MC, Le Toumelin G, Criollo A, Rain JC, Gautier F, Juin P et al. Functional and physical interaction between $\mathrm{Bcl}-\mathrm{X}(\mathrm{L})$ and a BH3-like domain in Beclin-1. EMBO J 2007; 26: 2527-2539.

22. Balaburski GM, Hontz RD, Murphy ME. p53 and ARF: unexpected players in autophagy Trends Cell Biol 2010; 20: 363-369.

23. Tasdemir E, Maiuri MC, Galluzzi L, Vitale I, Djavaheri-Mergny M, D'Amelio M et al. Regulation of autophagy by cytoplasmic p53. Nat Cell Biol 2008; 10: 676-687.

24. Mathew R, Karp CM, Beaudoin B, Vuong N, Chen G, Chen HY et al. Autophagy suppresses tumorigenesis through elimination of p62. Cell 2009; 137: 1062-1075.

25. Kondo Y, Kanzawa T, Sawaya R, Kondo S. The role of autophagy in cancer development and response to therapy. Nat Rev Cancer 2005; 5: 726-734.

26. Xu ZX, Liang J, Haridas V, Gaikwad A, Connolly FP, Mills GB et al. A plant triterpenoid avicin $\mathrm{D}$, induces autophagy by activation of AMP-activated protein kinase. Cell Death Differ 2007; 14: 1948-1957.

27. Raj L, Ide T, Gurkar AU, Foley M, Schenone M, Li X et al. Selective killing of cancer cells by a small molecule targeting the stress response to ROS. Nature 2011; 475: 231-234.

28. Burgess DJ. Anticancer drugs: selective oxycution? Nat Rev Drug Discov 2011; 10: 658

29. Adams DJ, Dai M, Pellegrino G, Wagner BK, Stern AM, Shamji AF et al. Synthesis, cellular evaluation, and mechanism of action of piperlongumine analogs. Proc Natl Acad Sci USA 2012; 109: 15115-15120.

30. Zhang DW, Shao J, Lin J, Zhang N, Lu BJ, Lin SC et al. RIP3, an energy metabolism regulator that switches TNF-induced cell death from apoptosis to necrosis. Science 2009; 325: 332-336.

31. Wang Z, Jiang H, Chen S, Du F, Wang X. The mitochondrial phosphatase PGAM5 functions at the convergence point of multiple necrotic death pathways. Cell 2012; 148: 228-243.

32. Degterev A, Hitomi J, Germscheid M, Ch'en IL, Korkina O, Teng X et al. Identification of RIP1 kinase as a specific cellular target of necrostatins. Nat Chem Biol 2008; 4 313-321.
33. Liang J, Shao SH, Xu ZX, Hennessy B, Ding Z, Larrea M et al. The energy sensing LKB1-AMPK pathway regulates p27(kip1) phosphorylation mediating the decision to enter autophagy or apoptosis. Nat Cell Biol 2007; 9: 218-224.

34. Golovine KV, Makhov PB, Teper E, Kutikov A, Canter D, Uzzo RG et al. Piperlongumine induces rapid depletion of the androgen receptor in human prostate cancer cells. Prostate 2013; 73: 23-30.

35. Dolado I, Swat A, Ajenjo N, De Vita G, Cuadrado A, Nebreda AR. p38alpha MAP kinase as a sensor of reactive oxygen species in tumorigenesis. Cancer Cell 2007; 11: 191-205.

36. Gutierrez-Uzquiza A, Arechederra M, Bragado P, Aguirre-Ghiso JA, Porras A. p38alpha mediates cell survival in response to oxidative stress via induction of antioxidant genes: effect on the p70S6K pathway. J Biol Chem 2012; 287: 2632-2642.

37. Hsieh CC, Kuro-o M, Rosenblatt KP, Brobey R, Papaconstantinou J. The ASK1Signalosome regulates p38 MAPK activity in response to levels of endogenous oxidative stress in the Klotho mouse models of aging. Aging (Albany NY) 2010; 2: 597-611.

38. Chen Y, McMillan-Ward E, Kong J, Israels SJ, Gibson SB. Mitochondrial electrontransport-chain inhibitors of complexes I and II induce autophagic cell death mediated by reactive oxygen species. J Cell Sci 2007; 120(Pt 23): 4155-4166.

39. Xiao D, Powolny AA, Moura MB, Kelley EE, Bommareddy A, Kim SH et al. Phenethyl isothiocyanate inhibits oxidative phosphorylation to trigger reactive oxygen speciesmediated death of human prostate cancer cells. J Biol Chem 2010; 285: 26558-26569.

40. Xiao D, Johnson CS, Trump DL, Singh SV. Proteasome-mediated degradation of cell division cycle $25 \mathrm{C}$ and cyclin-dependent kinase 1 in phenethyl isothiocyanate-induced G2-M-phase cell cycle arrest in PC-3 human prostate cancer cells. Mol Cancer Ther 2004; 3: $567-575$

41. Zhan Y, Gong K, Chen C, Wang H, Li W. P38 MAP kinase functions as a switch in MS-275induced reactive oxygen species-dependent autophagy and apoptosis in human colon cancer cells. Free Radic Biol Med 2012; 53: 532-543.

42. Moruno-Manchon JF, Perez-Jimenez E, Knecht E. Glucose induces autophagy under starvation conditions by a p38 MAPK-dependent pathway. Biochem J 2013; 449: 497-506.

43. de la Cruz-Morcillo MA, Valero ML, Callejas-Valera JL, Arias-Gonzalez L, Melgar-Rojas P, Galan-Moya EM et al. P38MAPK is a major determinant of the balance between apoptosis and autophagy triggered by 5 -fluorouracil: implication in resistance. Oncogene 2012; 31: 1073-1085.

44. Cruz-Morcillo MA, Sanchez-Prieto R. Autop38-phagy and apop38-tosis in genotoxic stress: a strange duo. Autophagy 2012; 8: 135-137.

45. Keil E, Hocker R, Schuster M, Essmann F, Ueffing N, Hoffman B et al. Phosphorylation of Atg5 by the Gadd45beta-MEKK4-p38 pathway inhibits autophagy. Cell Death Differ 2013; 20: $321-332$.

46. Wang $Y$, Zhang $Y$, Yoneyama $H$, Onai $N$, Sato $T$, Matsushima $K$. Identification of CD8alpha + CD11c- lineage phenotype-negative cells in the spleen as committed precursor of CD8alpha + dendritic cells. Blood 2002; 100: 569-577.

(c) (i) () $\Theta$ Cell Death and Disease is an open-access journal published by Nature Publishing Group. This work is licensed under a Creative Commons Attribution-NonCommercialNoDerivs 3.0 Unported License. To view a copy of this license, visit http://creativecommons.org/licenses/by-nc-nd/3.0/

\section{Supplementary Information accompanies this paper on Cell Death and Disease website (http://www.nature.com/cddis)}

